\title{
Design of Background Noise Suppression Circuit for Mobile Phones
}

\author{
Peng Luo \\ College of Electronical Information Engineering, West China Normal University, Nanchong, China \\ Email: 20468323@qq.com
}

Received 15 May 2016; accepted 21 July 2016; published 26 July 2016

Copyright (C) 2016 by author and Scientific Research Publishing Inc. This work is licensed under the Creative Commons Attribution International License (CC BY). http://creativecommons.org/licenses/by/4.0/

(c) () D Den Access

\begin{abstract}
With the purpose of reducing the influence of background noise on the call quality of mobile phones, background noise suppression circuit is designed based on the principle of self-adaptive noise cancellation. Because this method is not involved in the nature of the noise itself, it can be used both for stationary noise cancellation and quasi-stationary noise cancellation. The working principle and circuit design of the system are introduced in detail. Simulated experiment was conducted in the lab, and its experimental results were analyzed. The experimental results show that the circuit works well with low cost, and has a broad prospect of application and popularization.
\end{abstract}

\section{Keywords}

Mobile Phones, Noise Suppression, Self-Adaptive Noise Cancellation, AD623

\section{Introduction}

As we know, the most basic function of mobile phones is to realize the voice communication, no matter how strong the functions of the mobile phones are. In crowded situations, however, listeners hear not only the addressor's voice, but various kinds of voice else (often referred to as background noise). Background noise can interfere with or even cover the speaker's voice, thereby causes difficulties or even failures of voice communication. Therefore, the study of background noise suppression technology has very important practical significance.

Background noise suppression techniques mainly include [1]: noise proof microphone technology, headphone technology, compandor technology and digital signal processing technology. Forte media adopt Advanced Microphone Array Processing (AMAP) technique in the anti-noise microphone technology [2], in which nonlinear acoustic echo cancellation technology can achieve fully anechoic communication effect. Through the sensor and 
DSP, Aliph in the United States achieves the goal of eliminating noise and improving call quality for the Jawbone headset [3]. The compandor technology is not only to compress the call signal but also to reduce the external noise when the signal is transmitted. However, when the signal is received, the compandor technology is not only to expand the call signal but also to reduce the background noise. The nature of noise suppression is filter, which is one of the main contents of the digital signal processing. Then it is logical to suppress noise by using digital signal processing technology. With the speed being faster and the price being cheaper of digital signal processor, digital signal processing technology has become the main technology of background noise suppression.

\section{Research Question}

The digital technique includes spectral subtraction, wavelet analysis and adaptive filter, and so on [4]. The spectral subtraction method has the advantages of less computation and better noise cancellation, but it also has the disadvantages of residual music noise Wavelet analysis denoising method is better in the auditory effect and endpoint information retention, but it will suppress some useful high frequency component as the high frequency noise [5]. Because the adaptive filter is not involved in the nature of the noise itself, it can be used both for stationary noise cancellation and quasi-stationary noise cancellation. Moreover, the adaptive filter of the digital signal processing technology has been widely used for its excellent self-learning and auto tracking [6].

With the environment changing, the parameters of the adaptive filter can be changed by the adaptive algorithm. Usually, the adaptive filter is realized by DSP, FPGA and other hardware technology. At this time, the effect of noise elimination is better, but the cost is higher. Therefore, the background noise suppression circuit was designed in the paper through analog circuit, which has the advantages of simple circuit and low cost. The circuit is based on the principle of noise cancellation (the deformation of adaptive filter theory), so it eliminates the background noise better.

\section{Research Methodology}

\subsection{Working Principle}

The principle of self-adaptive noise cancellation is as follow. There are two sensors including primary sensor and reference sensor in Figure 1. In addition to accepting useful signal and outputting $s(n)$, primary sensor also accepts noise signal and output $n(n)$, namely its output $d(n)=s(n)+n(n)$; reference sensor accepts only noise signal and output $n_{1}(n)$ with certain correlation to $n(n)$. Under the action of the self-adaptive filter, $n_{1}(n)$ turns out $n^{\prime}(n) \approx n(n)$. Finally, through the subtracter, system output $z(n)=d(n)-n^{\prime}(n)=s(n)$. Obviously, the noise component $n(n)$ of the primary path has been offset [7].

\subsection{Circuit Design}

\subsubsection{Global Circuit}

Two microphones (primary MIC and reference MIC) are used to achieve the goal of noise reduction and denoising in the project (Figure 2). The primary MIC accepts the speaker's voice (useful signal) and background noise and turns them into electrical signal. The signal, after a band pass filter's processing, inputs to the in-phase input of instrumentation amplifier. At the same time, the reference MIC only accepts the background noise and turns them into electrical signal. The electrical signal inputs into the anti-phase input of the instrumentation

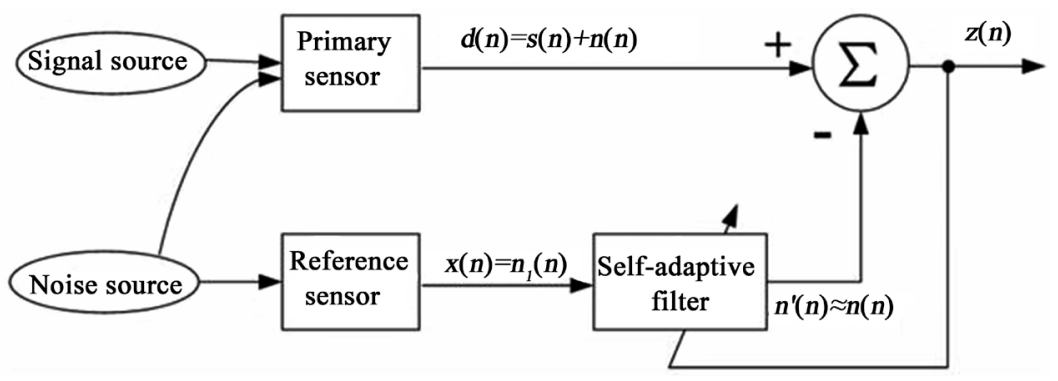

Figure 1. The principle of self-adaptive noise cancellation. 


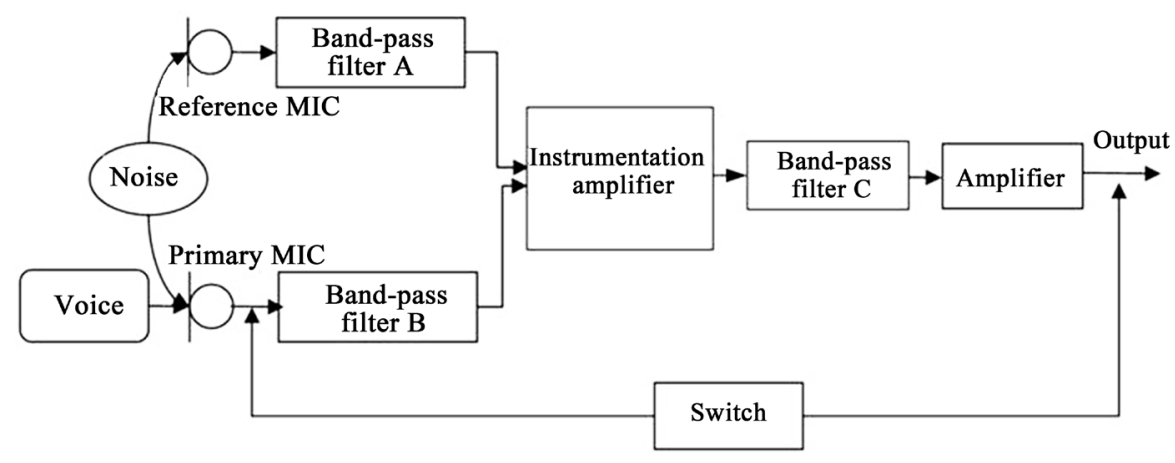

Figure 2. Block diagram of circuits.

amplifier through band-pass filter B. The two MICs are closer to each other, therefore they accept exactly the same background noise. Besides, the two MICs are the same, so the electrical signals converted from the noise through them are the same as well. Meanwhile, the band-pass filter A \& B are also the same, which ensures that two electrical signals corresponding to noise and inputting to the instrumentation amplifier are the same completely. Only in this way, can it be offset via the instrumentation amplifier, remaining electronic signal corresponding to the sound of voice. Electronic signal corresponding to voice outputs through band-pass filter C. In addition, through the amplifying circuit and switching circuit, this circuit can be connected to the mobile phone.

\subsubsection{Band-Pass Filter [8]}

Because the input sounds include not only the sound waves ( $\mathrm{f}=20 \mathrm{~Hz} \sim 20 \mathrm{kHz}$ ) people can hear, but also ultrasonic and infrasonic wave people cannot hear. Among which the latter is noise, we can remove them through band-pass filter (Figure 3).

\subsubsection{Instrumentation Amplifier [9] [10]}

To eliminate background noise, the filtered signal corresponding to reference MIC must be subtracted from the filtered signal corresponding to primary MIC. In this way, the noise counteract each other but voice (namely useful signal). It can be fulfilled through a differential amplifier AD623 (Figure 4). A $1.02 \mathrm{k} \Omega$ resistor is connected between pin 1 and pin 8 of AD623AN, which can achieve the subtraction of noise signal and amplificate 100 times for subtracted signal.

\section{Experiment and Result Analysis}

The virtual digital oscilloscope VS5202D, with two analog inputs CH1 \& CH2, was used in the experiment [11] [12]. CH1 was connected to the in-phase input of the instrument amplifier, measuring the output waveform of the primary microphone. $\mathrm{CH} 2$ was connected to the output of the whole circuit, measuring the output waveform of the whole circuit.

First talking to the primary microphone in quiet situation, the waveform of voice was measured as shown in Figure 5(a). Then making some background noise by playing a piece of music, the output waveform $\mathrm{CH} 1$ of the primary microphone was measured as shown in Figure 5(b). As a consequence, the primary MIC waveform and the voice waveform are quite different due to the presence of background noise. However, the whole circuit waveform i.e. $\mathrm{CH} 2$ in Figure 5(b) is basically in line with the voice waveform. This shows that the design circuit can really eliminate most of the background noise.

\section{Conclusion}

According to the background noise suppression technology, circuit for eliminating background noise of mobile phone was designed in this paper. And through experiments, it is proved that the circuit does have the function of eliminating most of the background noise. The reason for the failure to completely eliminate the noise is as follow: because of the limitation of the actual electronic process, two electrical signals, which correspond to the background noise of AD623's in-phase input and the anti-phase input, can't be exactly the same. In addition, the size of circuit is large, so it will significantly increase the volume and weight of the phone when uses in the 


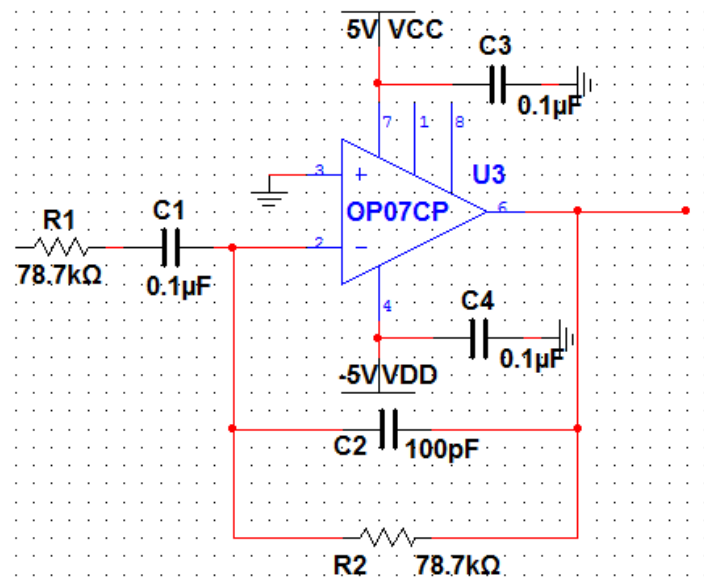

Figure 3. Band-pass filter.

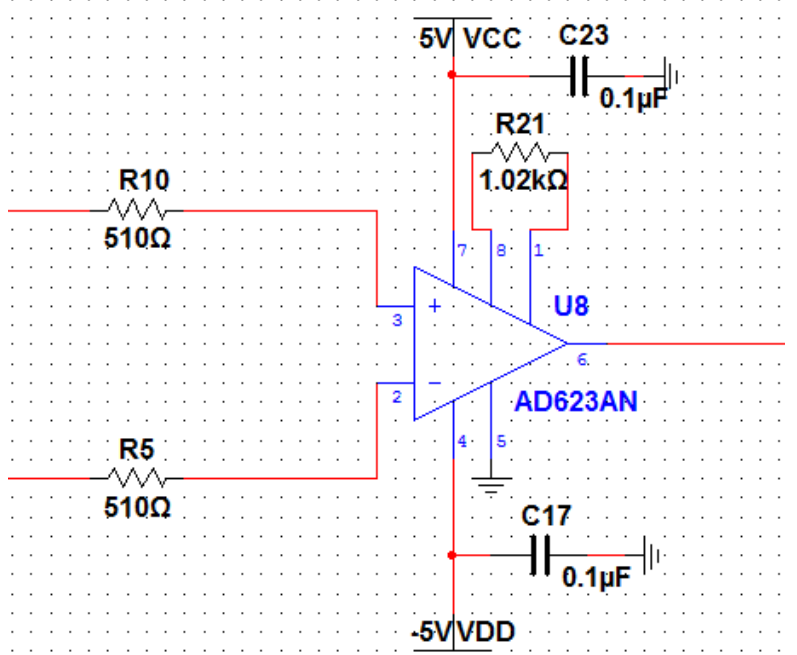

Figure 4. Instrumentation amplifier.

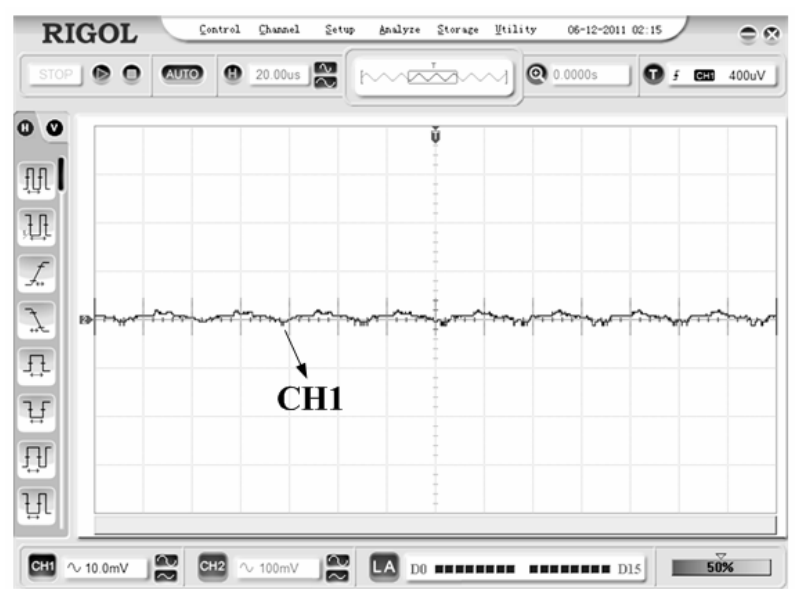

(a)

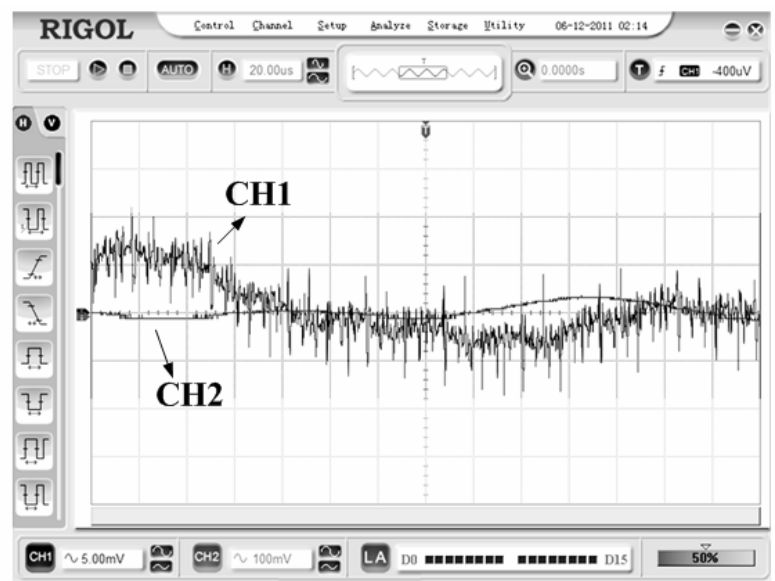

(b)

Figure 5. Display waveform of oscilloscope. (a) The waveform without background noise; (b) The waveform with background noise. 
mobile phones. Therefore, it should be make some improvements in the production process and integration of the circuit in the future, so that the circuit can really be used in the mobile phone.

\section{References}

[1] Hao, Y.C. (2007) Research and Simulation of Background Noise Cancellation to Mobile Phones. Master Dissertation, Jilin University, Changchun.

[2] Fortemedia, Inc. (2016) Fortemedia’s Updated FM1388 Series Provides Superior Voice Processing Solutions for Apple Carplay and ITU P.1100/P.1110 Compliance. http://www.fortemedia.com.cn/press/PR_160415.html

[3] TMC News (2008) CSR's Industry-Leading Blue Core Silicon inside Aliph's New Jawbone Bluetooth Headset. http://www.tmcnet.com/usubmit/2008/10/27/3735012.htm

[4] Li, Y.W. (2015) Hilbert-Huang Transform in the Application of Noisy Speech Processing. Master Dissertation, Heilongjiang University, Harbin.

[5] Tian, Y.J., Zuo, H.W. and Guo, F.H. (2008) Speech De-Noising Application Study. Technology Acoustic, 4, $557-561$.

[6] Da, X.L. (2009) Research and Implementation of Speech Enhancement on FPGA. Master Dissertation, Xihua University, Chengdu.

[7] Shao, C.H. (2008) The Analysis and Research of the Adaptive Noise Cancellation Method in Communication. Master Dissertation, Anhui University of Science \& Technology, Huainan.

[8] Matsui Bond (2006)100 Cases for OP Amplifier Application Technique. Science Press, Beijing.

[9] Yang, X.H., Hao, X.H. and Liu, M. (2001) A New Type of Integration Instrument Amplifier and Its Applications. Control and Instruments in Chemical Industry, 28, 56-57.

[10] Gao, G.T. (1995) Instrumentation Amplifier Application Technology. Science Press, Beijing.

[11] Li, W. (2008) The Use and Detection Techniques of Oscilloscope. Chemical Industry Press, Beijing.

[12] Bhunia, C., Giri, S., Kar, S., Haldar, S. and Purkait, P. (2004) A Low-Cost PC-Based Virtual Oscilloscope. IEEE Transactions on Education, 47, 295-299. http://dx.doi.org/10.1109/TE.2004.825527

\section{Submit or recommend next manuscript to SCIRP and we will provide best service for you:}

Accepting pre-submission inquiries through Email, Facebook, LinkedIn, Twitter, etc.

A wide selection of journals (inclusive of 9 subjects, more than 200 journals)

Providing 24-hour high-quality service

User-friendly online submission system

Fair and swift peer-review system

Efficient typesetting and proofreading procedure

Display of the result of downloads and visits, as well as the number of cited articles

Maximum dissemination of your research work

Submit your manuscript at: http://papersubmission.scirp.org/ 